\title{
Kinetic Measurements of Cell Surface E-Selectin/Carbohydrate Ligand Interactions
}

\author{
Mian Long, ${ }^{1,2,3}$ Hong ZhaO, ${ }^{1}$ KuO-Sen Huang, ${ }^{4}$ and Cheng Zhu ${ }^{1}$ \\ ${ }^{1}$ George W. Woodruff School of Mechanical Engineering and Wallace H. Coulter Department of Biomedical Engineering, Georgia \\ Institute of Technology, Atlanta, GA, ${ }^{2}$ College of Bioengineering, Chongqing University, Chongqing $400044,{ }^{3} \mathrm{National}$ \\ Microgravity Laboratory, Institute of Mechanics, Chinese Academy of Science, Beijing 100080, People's Republic \\ of China, and ${ }^{4}$ Department of Discovery Technologies, Hoffmann-La Roche Inc., Nutley, NJ
}

(Received 9 April 2001; accepted 7 September 2001)

\begin{abstract}
Selectin/ligand interactions initiate the multistep adhesion and signaling cascades in the recruitment of leukocytes from circulation to inflamed tissues and may also play a role in tumor metastasis. Kinetic properties of these interactions are essential determinants governing blood-borne cells' tethering to and rolling on the vessel wall. Extending our recently developed micropipette method, we have measured the kinetic rates of E-selectin/ligand interactions. Red cells coated with an E-selectin construct were allowed to bind HL-60 or Colo-205 cells bearing carbohydrate ligands. Specific adhesions were observed to occur at isolated points, the frequency of which followed a Poisson distribution. These point attachments were formed at the same rate with both the HL-60 and Colo-205 cells $\left(0.14 \pm 0.04\right.$ and $0.13 \pm 0.03 \mu \mathrm{m}^{2} \mathrm{~s}^{-1}$ per unit density of E-selectin, respectively) but dissociated from the former at a rate twice as fast as did from the latter $(0.92 \pm 0.23$ and 0.44 $\pm 0.10 \mathrm{~s}^{-1}$, respectively). The reverse rates agree well with those measured by the flow chamber. The forward rates are orders of magnitude higher than those of $\mathrm{Fc} \gamma$ receptors interacting with IgG measured under similar conditions, consistent with the rapid kinetics requirement for the function of E-selectin/ligand binding, which is to capture leukocytes on endothelial surfaces from flow. (C) 2001 Biomedical Engineering Society. [DOI: 10.1114/1.1415529]
\end{abstract}

Keywords-Micropipette, Single-bond adhesion, Probabilistic model, Reaction rate, Dissociation constant.

\section{INTRODUCTION}

E-selectin is a member of the selectin family of adhesion molecules that are important in processes such as leukocyte trafficking, thrombosis, inflammation, and ischemia reperfusion. ${ }^{19,32}$ It consists of an $\mathrm{N}$-terminal, calcium-type lectin (Lec) domain, followed by an epidermal growth factor (EGF)-like module, multiple copies of

Address correspondence and reprint requests to Dr. Cheng Zhu, George W. Woodruff School of Mechanical Engineering and Wallace H. Coulter Department of Biomedical Engineering, Georgia Institute of Technology, Atlanta, GA 30332. Electronic mail: cheng.zhu@ me.gatech.edu

Hong Zhao is on leave from Clinical Research Institute, ChinaJapan Friendship Hospital, Beijing 100029, P. R. China. consensus repeat units characteristic of complement binding proteins, a transmembrane segment, and a short cytoplasmic domain. Inducible on stimulated endothelial cells, E-selectin binds carbohydrate ligands expressed on leukocytes, including E-selectin ligand 1 (ESL-1), ${ }^{16,44}$ and other glycoproteins decorated with oligosaccharides. ${ }^{4,49,60}$ The specific carbohydrate structures that are recognized by E-selectin include modifications of the sialylated blood groups sialyl-Lewis a $\left(\mathrm{sLe}^{a}\right)$ and sialyl-Lewis $x\left(\mathrm{sLe}^{x}\right){ }^{51,52}$ Selectin/ligand interactions mediate the first step in the multistep adhesion and signaling cascades involved in the trafficking of lymphocytes into secondary lymphoid tissues and the recruitment of leukocytes from the circulation to sites of infection or injury. ${ }^{17,25,43}$ Furthermore, high levels of $\mathrm{sLe}^{a}$ and $\mathrm{sLe}^{x}$ epitopes are expressed in some carcinoma cells, such as Colo-205, which have been shown to mediate adhesion of these tumor cells to endothelial cells via E-selectin. ${ }^{24,46,49,57}$ This and other observations suggest a role of E-selectin/ligand interaction in tumor metastasis. $^{3,7}$

Kinetic rate and binding affinity constants are essential determinants of cell adhesion, as these parameters govern how likely, how rapidly, and how strongly cells bind, how long they remain bound, as well as how many bonds will be formed. Perhaps there is no better example than the selectin/ligand interactions in this regard, for their specialized function is to mediate flowing cells to tether to and to roll on the blood vessel wall. ${ }^{9}$ Rolling adhesion is distinct from the firm adhesion under static conditions mediated by integrins in the second step of the adhesion and signaling processes. ${ }^{20,53}$ The ability for the selectins to mediate cell tethering and rolling is believed to be due, at least in part, to their rapid kinetics. It has been hypothesized that fast forward rates are required to enable selectin/ligand interactions to effectively capture leukocytes on edothelial surface from flow. The strict conditions under which selectins have to do such a job can be better appreciated from the following consid- 
erations: In postcapillary venules where selectinmediated cell tethering and rolling occur, the leukocyte travels in the blood stream at a millimeter per second velocity. This may seem slow in the macroscopic world, but compared to the $10 \mu \mathrm{m}$ cell size it is a speed of 100 cell diam per second. For a macroscopic object, e.g., a car, to move 100 times of its size per second requires a speed of hundreds of miles per hour. A direct consequence of the rapid relative motions is the brief encounter time when the flowing cell collides with the stationary vascular wall, which is millisecond. To bind once every 100 collisions requires a cellular forward rate of 10 per second. For activated endothelial cells that express on their surface 370 E-selectin per micron square, this translates to a per E-selectin density forward rate of $0.027 \mu \mathrm{m}^{2} \mathrm{~s}^{-1}$. This number is orders of magnitude larger than the per receptor density forward rates of $\mathrm{Fc} \gamma$ receptors interacting with immunoglobulin $G$ $(\mathrm{IgG}),{ }^{12,13,54-56}$ but, as will be shown later, it is consistent with values measured in this work.

The interest in kinetics is reflected by the rapidly increasing use of optical biosensors to measure kinetic rates of adhesion molecules. Such measurements have indeed shown fast kinetics for ligand binding of P- and L-selectins compared to typical antibody/antigen interactions. $^{27,31}$ However, the relevance of these socalled three-dimensional, or $3 \mathrm{D}$, kinetic rates, measured with soluble ligands binding to surface-linked receptors, to cell adhesion is still in question. It has long been argued that the 3D parameters should be quite different from their two-dimensional (2D) counterparts, namely, those of binding between membrane-bound molecules. ${ }^{5,6}$ Indeed, the 2D forward-rate $k_{f}$ (in $\mu \mathrm{m}^{2} \mathrm{~s}^{-1}$ ) and binding affinity $K_{a}$ (in $\mu \mathrm{m}^{2}$ ) have different dimensions, and hence, are physically distinct properties, from their $3 \mathrm{D}$ counterparts (in $\mathrm{M}^{-1} \mathrm{~s}^{-1}$ and $\mathrm{M}^{-1}$, respectively).

Kaplanski et al. were the first to measure 2D kinetics using the flow chamber technique. ${ }^{18}$ Since then, there have been two other flow chamber ${ }^{2,42}$ and microcantilever ${ }^{47}$ measurements on the dependence of reverse rate $k_{r}$ (in $\mathrm{s}^{-1}$ ) on force $f$ for E-selectin/ carbohydrate ligand interactions. However, there are two issues in the flow chamber studies that have yet to be addressed. In the limited studies where forward-rate measurements were attempted, ${ }^{18,35,36,45}$ it was difficult to dissect $k_{f}$ because the measured binding rate of flowing cells to the chamber floor includes effects of collision frequency and encounter duration, none of which can be separately measured by independent experiments. Second, the zero-force reverse rate was not directly measured; rather, it was estimated by extrapolating the $k_{r}$ vs $f$ data to $f=0$.

To address these issues, we recently developed a micropipette method that allows for 2D measurement of both forward- and reverse-rate constants in the absence of tensile force. ${ }^{12}$ In the micropipette, many conditions that cannot be controlled in the flow chamber are staged by the experimenter. These include whether, when, and how a contact will occur, how long a time and how large an area the two cells will be in contact, and when they will be separated at what speed and by how large a force. Thus, the collision frequency and encounter duration are directly controlled by the experimenter. Also, the reverse rate is evaluated at $f=0$ because it is estimated from how fast bond dissociation counterbalances bond formation in the absence of force, as opposed to how long a bond lasts when it is subject to a force. This method has been successfully used in several studies to measure 2D kinetics of $\mathrm{Fc} \gamma$ receptors $(\mathrm{Fc} \gamma \mathrm{R})$ interacting with their IgG ligands. ${ }^{12,13,54-56}$

In this work, we have extended our micropipette method in two areas. First, the method was applied to measure the 2D kinetics of a biological system that had not been tested by the micropipette but extensively studied by the flow chamber., ${ }^{2,42}$ A recombinant E-selectin construct consisting of the Lec-EGF fragment, which is known to be the minimum functional unit for ligand binding, ${ }^{21}$ was coated onto the surface of human red blood cells (RBC) by a capture monoclonal antibody $(\mathrm{mAb})$. This was allowed to interact with carbohydrate ligands expressed on a human promyelocytic leukemia cell line (HL-60) or a human colon adenocarcinoma cell line (Colo-205). They were so chosen because the former serves as a model for leukocytes and the latter represents carcinoma cells. Second, we attempted to quantify the probability of having individual bonds instead of merely measuring the probability of adhesion. Direct visualization of the contact area between the two interacting cells during their separation enabled us to segregate the adhesions into subgroups according to the number of point attachments linking the two membranes. The frequencies of these point attachments were found to follow the Poisson distribution. These observations validate the use of McQuarrie's small-system kinetics formulation. ${ }^{26}$

The reverse rates measured by the micropipette in this study were compared to those estimated from the flow chamber, $2,18,42$ which showed good agreement supporting the validity of both methods. By comparison, the forward rates obtained here represent new information, since the flow chamber has been unable to measure this parameter. The forward rates are found to be orders of magnitude higher than those of Fc $\gamma$ Rs interacting with $\mathrm{IgG}$ measured under similar conditions, ${ }^{12,13,54-56}$ consistent with the rapid kinetics requirement for the function of E-selectin/ligand binding, which is to capture leukocytes on endothelial surfaces from flow. 


\section{MATERIALS AND METHODS}

Selectin Constructs, Antibodies, and Cell Lines

The soluble E-selectin construct consisting of only the Lec-EGF domains, ${ }^{21}$ and the anti-E-selectin nonblocking mAb 1D6 (mIgG1), ${ }^{15}$ were from Hoffmann-La Roche Inc. (Nutley, NJ). The P-selectin Lec-EGF construct, ${ }^{28}$ anti-E-selectin blocking (ES1) and nonblocking (ES2) mAbs (both mIgG1), ${ }^{33}$ as well as anti-P-selectin glycoprotein ligand 1 (PSGL-1) blocking mAb PL1 (mIgG1) ${ }^{30}$ were generous gifts from Dr. R. P. McEver (University of Oklahoma, Oklahoma City, OK). AntisLe $^{x}$ mAbs KM93 and CSLEX-1 (both mIgM) were obtained from Kamiya Biomedical Co. (Seattle, WA) and Becton Dickinson (San Jose, CA), respectively. Total human (h) IgG, rabbit antihuman IgG polyclonal antibody $\mathrm{F}\left(\mathrm{ab}^{\prime}\right)_{2}$, mouse $(\mathrm{m})$ IgG1, and $\mathrm{mIgM}$ control antibodies were from Sigma Chemical Co. (St. Louis, MO). Human colon adenocarcinoma cell line Colo-205 cells was from the American Type Culture Collection (Rockville, MD). Human promyelocytic leukemia cell line HL-60 was from Dr. R. P. McEver. Both cell lines were grown in RPMI 1640 medium (Sigma) supplemented with $2 \mathrm{mM}$ L-glutamine, $100 \mathrm{U} \mathrm{ml}^{-1}$ penicillin, $10 \mathrm{mg} \mathrm{ml}^{-1}$ streptomycin, $0.25 \mu \mathrm{g} \mathrm{ml}^{-1}$ amphotericin $\mathrm{B}$, and $10 \%$ fetal bovine serum. Our Chinese hamster ovary $(\mathrm{CHO})$ cells expressing $\mathrm{Fc} \gamma \mathrm{RIIIb}$ (NA2 allele) and their culturing method have been described. ${ }^{54}$

\section{Coupling Protein to RBCs}

A modified chromium chloride method, described in detailed previously, ${ }^{12,54}$ was used to couple the capture $\mathrm{mAb}$ (1D6) or isotype-matched irrelevant $\mathrm{mIgG}$ on the surfaces of RBCs. Briefly, RBCs were isolated from fresh whole blood of healthy donors by centrifugation through Histopaque 1119 (Sigma). Collected packed cells were stored in RBC storage solution (EAS45), ${ }^{14}$ or phosphate-buffered saline (PBS), at $4{ }^{\circ} \mathrm{C}$. For coupling, about $10^{8}$ cells were washed five times with $0.85 \%$ phosphate-free saline and resuspended in $250 \mu \mathrm{l}$ of saline solution. Antibody in phosphate-free saline was added to the cells, resulting in a final antibody concentration of $10 \mu \mathrm{g} \mathrm{ml}^{-1}$. Equal volume of $\mathrm{CrCl}_{3}$ solution, prepared by diluting at 1:400-1:1600 ratios aged $1 \%$ $\mathrm{CrCl}_{3}$ in $0.02 \mathrm{M}$ acetate buffer, was added dropwise to the cell suspension with continuous vortexing. After 5 min, $\mathrm{PBS} / 5 \mathrm{mM} \mathrm{EDTA} / 1 \% \mathrm{BSA}$ at a volume equal to that of the reaction mix were added to terminate the reaction. Antibody-coupled cells were rinsed twice and stored in EAS45 at $4{ }^{\circ} \mathrm{C}$ until use. Coupling efficiency was examined by flow cytometry, using as a standard CD58, which are constitutively expressed on RBC surface at a known density. ${ }^{40}$ Cells were incubated with 50-200 $\mathrm{ng} \mathrm{ml}^{-1}$ selectin constructs for $30 \mathrm{~min}$ at $4{ }^{\circ} \mathrm{C}$ before the micropipette experiment or site density measurement.

\section{Site Density Determination}

To determine site densities of E-selectin coated on the RBC surfaces, mAb ES1 was iodinated as described in Ushiyama et al. ${ }^{50}$ Briefly, antibodies and $\mathrm{Na}\left[{ }^{125} \mathrm{I}\right]$ (ICN Biomedical, Irvine, CA) at a ratio of $100 \mu \mathrm{g}$ of protein to $400 \mu \mathrm{Ci}$ of ${ }^{125} \mathrm{I}$ were added to an Iodogen-coated glass tube on ice. After $5 \mathrm{~min}$ of incubation, the reaction was terminated by adding tyrosine solution at a concentration of $0.4 \mathrm{mg} \mathrm{ml}^{-1}$. Free ${ }^{125} \mathrm{I}$ was removed by gel filtration through a Sephadex G-25 M column (PD-10, Pharmacia, Peapack, NJ) equilibrated in labeling buffer $(25 \mathrm{mM}$ HEPES, $116.25 \mathrm{mM} \mathrm{NaCl}$ at $\mathrm{pH}$ 7.4). The eluted volume fractions from the first peak of the radioactivity versus fraction number plot were pooled, and the protein concentration was determined with a Micro BCA protein assay kit (Pierce, Rockford, IL) using the unlabeled protein as a standard. The labeled protein was stored with $0.1 \%$ BSA at $4{ }^{\circ} \mathrm{C}$ until use. The specific activity of the labeled protein ranged from $2500-5000 \mathrm{cpm} \mathrm{ng}^{-1}$. The labeled protein was routinely $>90 \%$ trichloroacetic acid precipitable.

${ }^{125}$ I-labeled ES1 was incubated with $10^{7} \mathrm{RBCs}$ that were previously coated with 1D6 and E-selectin construct at a concentration of $0.5-4 \mu \mathrm{g} \mathrm{ml}^{-1}$ in $150 \mu \mathrm{l}$ of Hanks/HBSS, $1 \%$ IgG-free BSA solution on ice for 40 min. $100 \mu \mathrm{l}$ of cell suspension was carefully layered on the top of an oil cushion (1:9 of apiezon: $n$-butyl phthalate) and then spun at 13,000 rpm for 6 min to separate the cell-bound from unbound ${ }^{125} \mathrm{I}$-ES1. Cell pellets were collected and counted in a gamma counter. Preliminary experiments indicated that the binding of ${ }^{125} \mathrm{I}-\mathrm{ES} 1$ to E-selectin construct saturated at a concentration of 0.5 $\mu \mathrm{g} \mathrm{ml}^{-1}$. The site densities were calculated from the numbers of bound ES1 per cell assuming a monovalent binding of ${ }^{125}$ I-ES1 to E-selectin construct at a saturating concentration.

\section{Micropipette Assay}

Our micropipette adhesion frequency assay has been described. ${ }^{12,54}$ Briefly, a RBC gently aspirated by a micropipette of $\sim 2 \mu \mathrm{m}$ inner diameter was used as an ultrasensitive force transducer to detect adhesions mediated by E-selectin and carbohydrate ligands expressed on either a HL-60 or a Colo-205 target cell, aspirated by another micropipette on the opposite side (Fig. 1). The RBC was partially swelled using a low osmotic solution. The RBC pipette was driven by a computer-controlled piezoelectric actuator (Polytec PI, Auburn, MA) to make repeated contacts with the target cell that was held stationary. The contact duration was kept constant in each 


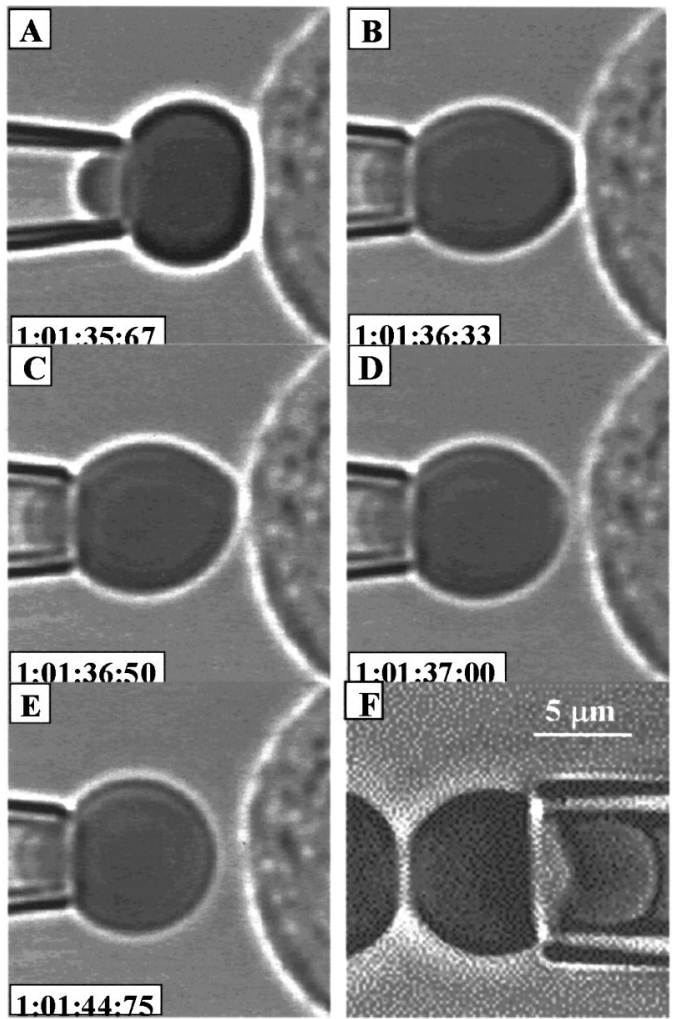

FIGURE 1. (A)-(E) Observation of adhesion mediated by isolated point attachments. A partially swelled RBC aspirated by a micropipette (left) was impinged onto a Colo-205 cell (right, only partially shown) to make a contact of an apparent area of $\sim 3 \mu \mathrm{m}^{2}$ (A). Upon retraction, the presence of an adhesion at the end of the contact duration was detected by the elongation of the RBC membrane, which was seen to be linked at three isolated points (B). The three point attachments were dissociated at different times, with the lower one being ruptured first (C), the upper one second (D), and the middle one last (E). The numbers shown are time in hour: minute:second:hundredth of a second. (F) Continuous attachment mediated by a large number of bonds. RBCs were coated with a high density of hlgG via $\mathrm{CrCl}_{3}$ method and were then incubated with a crosslinker-the rabbit antihuman IgG polyclonal antibody $F\left(a b^{\prime}\right)_{2}$. The left cell was aspirated with a high pressure to form a nearly rigid spherical surface outside the pipette mouth. The suction pressure in the right pipette was lower to allow its cell to form a large contact area when the two cells were impinged onto each other. Separation of the adherent cell pair was seen to occur as gradual peeling of the right cell's membrane. Bar $=5 \mu \mathrm{m}$ (see Ref. 61).

series of 100 contacts using a single pair of a RBC and a target cell but systematically varied over a range (0.5-16 s) using different cell pairs. The apparent contact area $\left(\sim 3 \mu \mathrm{m}^{2}\right)$ was kept constant throughout, as observed microscopically [Fig. 1(A)]. Adhesion, if present at the end of a controlled contact, was detected by the membrane deflection of the RBC during its retraction [Fig. 1(B)]. In addition, the number of isolated point attachments linking the two cell membranes was enumerated by direct observation during their rupture when the RBC was being retracted or held at a fixed distance of retraction [Figs. 1(B)-1(E)]. The images were observed using a Zeiss inverted microscope (Axiovert 100; Oberkochan, Germany) equipped with a $100 \times$ oil immersion 1.25 NA objective, a closed-circuit video system, and an image grabbing and analysis system. The probabilities of adhesion and of having $n(=0,1,2,3, \ldots)$ point attachments were estimated from the frequency of their occurrence. The suction pressure in the RBC pipette was controlled to be $1-5 \mathrm{~mm}$ of water height by a hydraulic pressure regulation system of an accuracy bet-

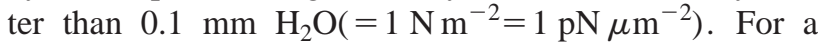
diameter of $\sim 6 \mu \mathrm{m}$ of the unaspirated portion of the $\mathrm{RBC}$, the force transducer has a spring constant of 10-50 $\mathrm{pN} \mu \mathrm{m}^{-1} .{ }^{11}$ With a better than $0.2 \mu \mathrm{m}$ spatial resolution for the RBC membrane deflection, this translates to a force detection sensitivity of better than $2 \mathrm{pN}$.

\section{RESULTS}

\section{Binding Was Specifically Mediated by E-Selectin/Ligand Bonds}

Results of experiments designed to address the binding specificity are shown in Fig. 2, which were performed in two ways. One directly compared binding frequencies measured at a given contact time $(t=5 \mathrm{~s})$ long enough for steady state to be achieved [Figs. 2(A) and 2(B)]. The other fitted binding frequencies measured in a range of contact times (0.5-16 s) to Eqs. (3) and (5) (see below) to extrapolate the steady-state $(t \rightarrow \infty)$ adhesion probabilities for comparison [Figs. 2(C) and 2(D)]. Upon incubation with E-selectin construct, the 1D6-coated RBCs adhered to Colo-205 cells in $\sim 50 \%$ of the contacts [Fig. 2(A)]. Adhesions were abolished when the E-selectin capture mAb 1D6 was replaced by an isotypematched irrelevant mIgG1 or when the E-selectin construct was substituted by the P-selectin construct. Adhesions were completely blocked by the anti-E-selectin blocking mAb (ES1), whereas the anti-E-selectin nonblocking mAb (ES2) had no effect [Fig. 2(B)]. Inclusion of EDTA $(5 \mathrm{mM})$ in the medium also abrogated adhesions, consistent with the known dependence of selectin/ carbohydrate ligand binding on divalent cations. ${ }^{22} \mathrm{Al}-$ though blocking effects were evident when compared to an isotype-matched irrelevant $\mathrm{mIgM}$, the anti-sLe ${ }^{x} \mathrm{mAbs}$ (KM93 and CSLEX-1) only partially inhibited binding. This might be due to the presence of other $\mathrm{sLe}^{x}$-related carbohydrate structures that bound E-selectin but were not blocked by KM93 and CSLEX-1. Similar results were obtained for RBCs coated with E-selectin construct interacting with HL-60 cells [Figs. 2(C) and 2(D)]. These data have established that the adhesions measured by the micropipette adhesion frequency assay were mediated by the specific interactions between the E-selectin construct and its ligands. 

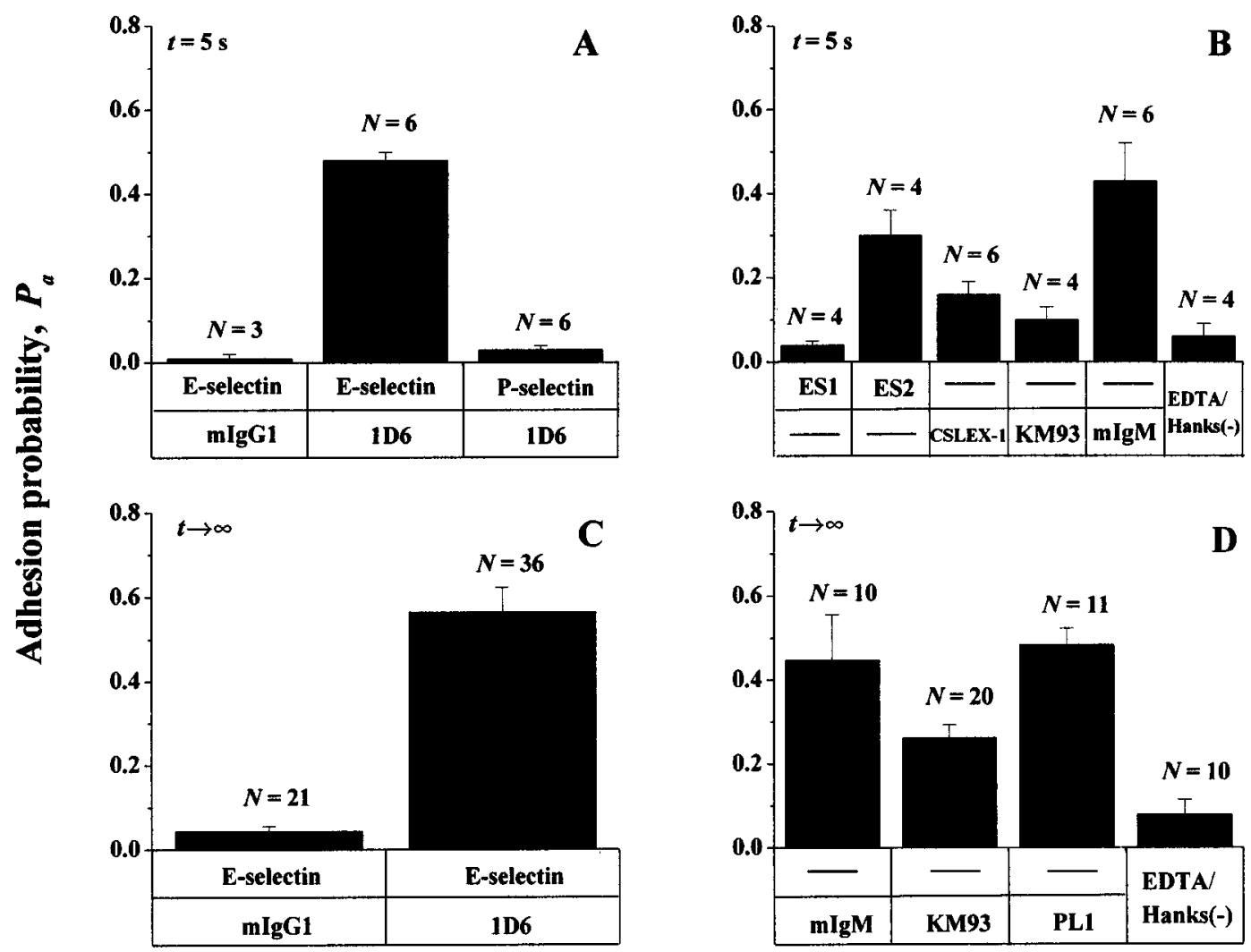

FIGURE 2. (A) and (C) Dependence of adhesion on E-selectin. (B) and (D) Inhibition of adhesion by blocking mAbs and by chelating the divalent cations. Data are presented as mean \pm standard error of the mean (SEM) of adhesion probability at a contact duration of $t=5 \mathrm{~s}(\mathrm{~A})$ and (B), or as adhesion probability extrapolated to steady state, $t \rightarrow \infty$, using Eqs. (3) and (5) to fit data measured in a range of contact times (C) and (D). $N$ values denote the numbers of cell pairs examined (each tested 100 times). (A) and (B) Colo-205 cell data. (C) and (D) HL-60 cell data.

\section{Point Attachments Followed Poisson Distribution}

The random occurrence and infrequency of adhesions in the micropipette assay suggest that they might be mediated by a low number of bonds. This contention is based on the assumption that forming any one bond is independent of and equivalent to the formation of other bonds. Under such a condition, the probability of having $n(=0,1,2, \ldots)$ bonds obeys a Poisson distribution: ${ }^{8,12}$

$$
p_{n}(\langle n\rangle)=\left(\langle n\rangle^{n} / n !\right) \exp (-\langle n\rangle)
$$

where the average number of bonds, $\langle n\rangle$, can be calculated either directly from the distribution, $p_{n}$,

$$
\langle n\rangle=\sum_{n=0}^{\infty} n p_{n},
$$

or by fitting Eq. (1) to the measured $p_{n}$. It follows from Eq. (1) that, when the probability of adhesion,

$$
P_{a} \equiv \sum_{n=1}^{\infty} p_{n}=1-\exp (-\langle n\rangle)
$$

is small, $P_{a} \approx\langle n\rangle$ and $p_{0} \gg p_{1} \gg p_{2} \gg p_{3} \ldots$, leading to the prediction that most adhesions are mediated by single-bond events. Although this probabilistic argument was used in a number of studies intended to measure single-bond adhesions, ${ }^{1,10,12,29,34,37,39,47}$ its underlying assumption had not been experimentally validated. Because the single-bond hypothesis is an important basis for the data interpretation in these studies, we have tested a prediction of this assumption, namely, the Poisson distribution of bonds.

Our test stems from the observation that, although the two cells were pressed against each other to make a controlled contact of large apparent area $\left[\sim 3 \mu \mathrm{m}^{2}\right.$, Fig. 1(A)], adhesions were only seen in a few (most often just 1) spatially separated distinct points, which detached sequentially. Although difficult to appreciate from the snap-shot images shown in Figs. 1(B)-1(E), the discrete and focal nature of this kind of "point attachments" can 
be clearly visualized by continuous video microscopy (a web movie is available, see Ref. 61) as rupture of a point attachment is always immediately followed by the rapid springing back of a large portion of the adherent RBC membrane to expose the remaining point attachments. This is in sharp contrast to another type, referred to as "area attachments," the separation of which required gradual peeling of the cell membrane from the edge of a seemingly continuously adhered area [Fig. 1(F)]. It seems reasonable to assume that the former attachments were mediated by a small number of discretely distributed bonds while the latter were mediated by a large number of continuously distributed bonds. Although we were unable to directly measure the number of molecular bonds in each of these point attachments or their precise dimension from the microscopic images alone, whether they are statistically independent and equivalent can be tested by examining the frequency distribution of their occurrence.

To conduct such a test, data pooled from experiments of many cell pairs (each tested 100 cycles to estimate an adhesion probability) were segregated into subgroups (bins) each of which has a narrow range of $P_{a}$, contains a similar number of adhesive events $(\sim 300)$, but includes different numbers of cell pairs (since cell pairs that produced higher $P_{a}$ resulted in more adhesive events). Because of the one-to-one relationship between $P_{a}$ and $\langle n\rangle$ [Eq. (3)], events in each subgroup are predicted to correspond to a narrow range of average number of point attachments. Adhesion tests resulted in no adhesion and adhesions via single, double, triple, etc., attachments in each subgroup were enumerated to determine their frequency distribution, $p_{n}$. The average number of point attachments, $\langle n\rangle$, was calculated either by fitting the Poisson distribution, Eq. (1), to the measured $p_{n}$ or by directly using the definition, Eq. (2), without any curve fitting. It was then compared to the theoretical $\langle n\rangle$ predicted from Eq. (3). It is evident from Fig. 3 that the measured, fitted, and predicted average number of point attachments compare reasonably well except when $\langle n\rangle$ approaches unity in the HL-60 case, where the measured and fitted $\langle n\rangle$ values are somewhat smaller than the theoretical $\langle n\rangle$ curve. This expected discrepancy will be discussed shortly.

For a more rigorous test, the experimentally measured $p_{n}$ vs $\langle n\rangle$ relationship was directly compared to the theoretical prediction based on Eq. (1). In such a comparison, not even a single curve-fitting parameter was used. As shown in Fig. 4, the frequencies of adhesions mediated by a given number of point attachments follow reasonably well the Poisson distribution over the range of $\langle n\rangle$ values tested, with respective coefficients of determination $R^{2}=0.707$ and 0.753 for the HL-60 [Fig. 4(A)] and Colo-205 [Fig. 4(B)] cases. Compared to those predicted by Eq. (1), the higher $p_{1}$ and the lower $p_{2}$ and

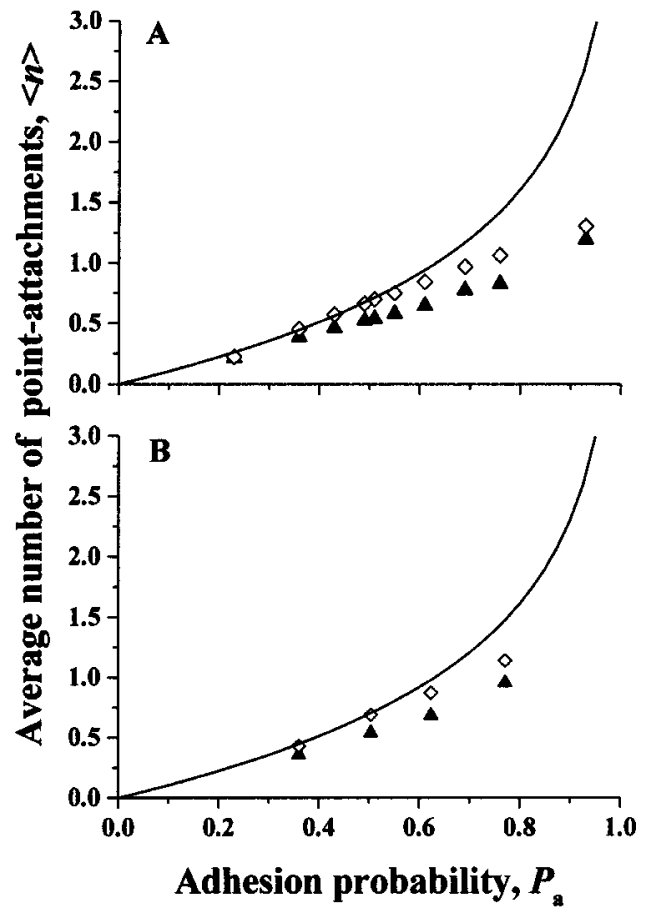

FIGURE 3. Comparison between measured $(\Delta)$, fitted $(\diamond)$, and predicted (curve) average numbers of point attachments, $\langle n\rangle$, as functions of the probability of adhesion, $P_{a}$ (average over a narrow range within each bin), for HL-60 (A) and Colo-205 (B) cells. The fitted $\langle n\rangle$ values were obtained by fitting the measured frequency distribution of point attachments, $p_{n}$, to the Poisson distribution, Eq. (1), and the theoretical prediction is based on Eq. (3). Bin size is $\sim 300$ adhesive events per bin for different number of cell pairs (3-23), each repeatedly tested 100 cycles to estimate the $P_{a}$ for that cell pair.

$p_{3}$ experimental values may be due to observation errors, which may also be the reason why the measured and fitted $\langle n\rangle$ values were lower than the predicted curve in Fig. 3. The determination of an adhesion to be a single, double, or triple point attachment was made from planar images such as those shown in Fig. 1, which projected the circular contact disk onto a line. As such, the observation tended to overestimate $p_{1}$ and underestimate $p_{2}$ and $p_{3}$, because multiple point attachments might project as a single point attachment from the side view. This might also bias the calculation of the average number of point attachments based on the measured $p_{n}$ towards a lower $\langle n\rangle$ value as seen in Fig. 3, especially at high values of $P_{a}$ where multiple point attachments are expected to be (and were observed to be) more frequent. Taken together, these data show that the point attachments were formed independently of each other and behaved as equivalent quantal binding units, and obeyed Poisson distributions over a $\langle n\rangle$ range of $0.1-1.2$. 


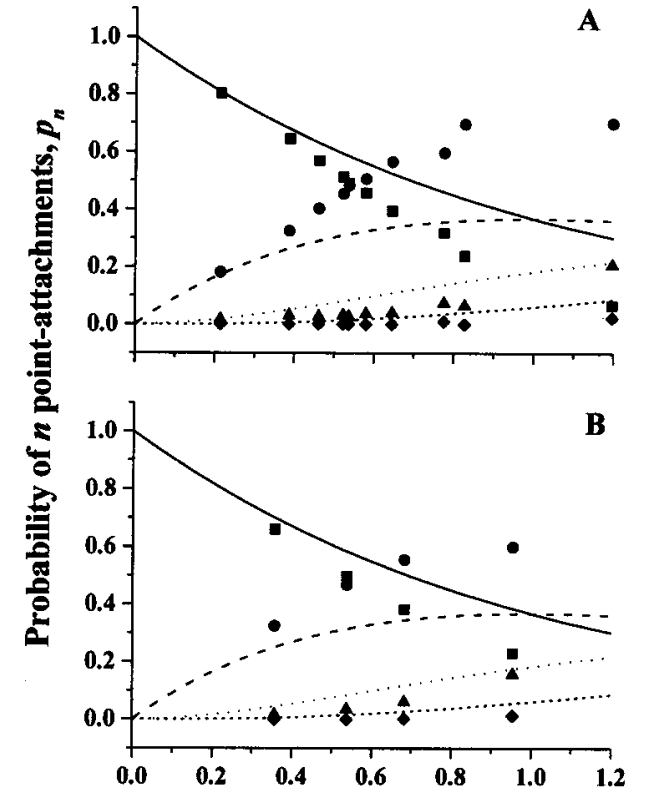

Average number of point-attachment, $<\boldsymbol{n}>$

FIGURE 4. Comparison between measured (points) and predicted (curves) probabilities of having zero ( $\square$, solid curve), one ( $\bullet$, long-dashed curve), two ( $\Delta$, dotted curve), and three $(\diamond$, short-dashed curve) point attachments as functions of the average number of point attachments for HL-60 (A) and Colo-205 (B) data. Theoretical predictions were calculated using Eq. (1).

\section{Average Point-Attachment Number Followed Simple Reversible Kinetics}

The average number of point attachments, $\langle n\rangle$, determined from Eq. (3) using the specific adhesion probability after removing the nonspecific binding, was systematically measured over a range of contact times $t(0.5-16$ s) for two or three E-selectin densities, $m_{r}$, for interactions with HL-60 and Colo-205 cells, respectively. The surface densities of E-selectin coated on RBCs were measured by independent experiments using radioimmunoassay as described in Materials and Methods. To examine the dependence of $\langle n\rangle$ on $m_{r}$, the ratio of $\langle n\rangle$ to $\left(m_{r}\right)^{v}$, where $v$ is an integer stoichiometric coefficient, was plotted against the contact time $t$. It was found that when $\nu=1$ is chosen, various $\langle n\rangle / m_{r}$ vs $t$ data sets corresponding to different $m_{r}$ values (indicated by the different symbols) collapse onto a single curve (Fig. 5), indicating that $\langle n\rangle$ was proportional to $m_{r}$. The $\langle n\rangle / m_{r}$ data in Fig. 5 increase with the contact time initially and then reach a plateau, and therefore contain both kinetic and equilibrium information.

For adhesive bonds (or bond clusters) formed independently and equivalently and distributed according to Eq. (1), their average number is predicted to follow a simple reversible kinetics: ${ }^{12}$

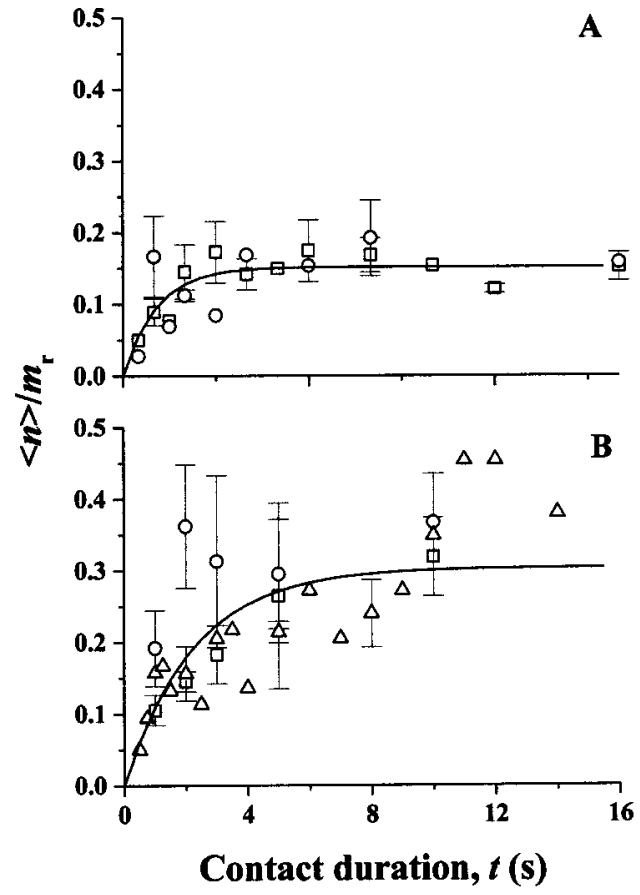

FIGURE 5. Dependence of average number of point attachments per E-selectin density, $\langle n\rangle / m_{r}$, on contact duration, $t$. Data (points) generated using different E-selectin densities are indicated by different symbols. The $m_{r}$ (in $\mu \mathrm{m}^{-2}$ ) values are $4.1(\bigcirc)$ and $5.2(\square)$ for the HL-60 data $(A)$ and $1.5(\bigcirc), 2.1$ $(\square)$, and $3.4(\triangle)$ for the Colo-205 data (B). Data are presented as mean $\pm S E M$ of $1-5$ pairs of cells of 100 cycles each. The total numbers of cell pairs tested are 49 and 63 for the HL-60 and Colo-205 data, respectively. Curves are theoretical fits to the data based on Eq. (5).

$$
d\langle n\rangle / d t=m_{r} m_{l} A_{c} k_{f}^{0}-k_{r}^{0}\langle n\rangle,
$$

where $k_{f}^{0}$ and $k_{r}^{0}$ are the respective forward- and reverserate constants, $m_{l}$ is the ligand density, and $A_{c}$ is the contact area. The superscript 0 emphasizes that these kinetic rates are evaluated at the zero-force state, for during the contact time the two cells were pressed against each other with no tensile force acting on the bonds. The zero initial condition solution of Eq. (4) is

$$
\langle n\rangle=m_{r} m_{l} A_{c} K_{a}^{0}\left[1-\exp \left(-k_{r}^{0} t\right)\right]
$$

where $K_{a}^{0} \equiv k_{f}^{0} / k_{r}^{0}$ is the equilibrium binding affinity. As shown in Fig. 5, Eq. (5) was found to globally fit reasonably well the measured average number of point attachments for two (HL-60) or three (Colo-205) E-selectin densities with a single set of parameters $\left(m_{l} A_{c} K_{a}^{0}\right.$ and $\left.k_{r}^{0}\right)$.

The effective affinity of the ligand presenting cell per unit density of E-selectin, $m_{l} A_{c} K_{a}^{0}$, can be estimated from plateau level of $\langle n\rangle / m_{r}$ whereas the reverse rate for dissociation, $k_{r}^{0}$, is related to the time $t_{1 / 2}$ required for 
TABLE 1. Summary of kinetic constants (mean \pm SEM).

\begin{tabular}{|l|cc|}
\hline Parameters Cells & HL-60 & Colo-205 \\
\hline$k_{r}^{0}, \mathrm{~s}^{-1}$ & $0.92 \pm 0.23$ & $0.44 \pm 0.10$ \\
$m_{1} A_{c} k_{f}^{0}, \mu \mathrm{m}^{2} \mathrm{~s}^{-1}$ & $0.14 \pm 0.04$ & $0.13 \pm 0.03$ \\
$m_{l} A_{c} K_{a}^{0}, \mu \mathrm{m}^{2}$ & $0.15 \pm 0.01$ & $0.30 \pm 0.03$ \\
\hline
\end{tabular}

$\langle n\rangle / m_{r}$ to achieve half of the plateau level, $k_{r}^{0}$ $=\ln 2 / t_{1 / 2}$. The initial slope, $d\left(\langle n\rangle / m_{r}\right) /\left.d t\right|_{t=0}$, is equal to the effective cellular forward-rate per unit density of E-selectin, $m_{l} A_{c} k_{f}^{0}$. The fitted parameters are summarized in Table 1 for the two cellular systems examined. Since neither were the precise structure(s) of carbohydrate ligands on the HL-60 and Colo-205 cells for E-selectin identified nor were their densities determined, we could only compare the E-selectin-mediated cellular kinetic rates. The data in Table 1 indicate that E-selectin coated RBCs formed point attachments with HL-60 and Colo-205 cells at the same rate but dissociated from HL-60 cells twice as fast as did from Colo-205 cells, giving an equilibrium binding level of the Colo-205 cells twice that of the HL-60 cells.

\section{DISCUSSION}

\section{Point Attachments Behaved as Quantal Binding Units}

In this work, we have extended our recently developed micropipette method for measuring kinetics of cellbound adhesion molecules. ${ }^{12}$ Previously, only binary outcomes (i.e., whether adhesion presented or not) were enumerated from micropipette adhesion tests, ${ }^{12,13,54-56}$ although the discrete and focal nature of these adhesions, i.e., that the two cell membranes were actually linked by spatially isolated point attachments, was noted. ${ }^{12}$ Here, the adhesions were further dissected into single, double, triple, etc., point attachments. It was found that these point attachments behaved as statistically independent and equivalent events that followed the Poisson distribution (Figs. 3 and 4). Their kinetics obeyed probabilistic kinetics formulation for small systems, ${ }^{26}$ as Eqs. (1) and (5) represent an approximate solution of McQuarrie's master equations with zero-force rate constants. ${ }^{12,23}$ These observations have provided direct physical evidence to support the hypothesis that small-scale, shortduration adhesions such as those quantified by the present micropipette assay were mediated by a low number of discrete, quantal binding units. In the absence of multimeric binding, these quantal binding units are most likely single bonds. ${ }^{59}$ As has been pointed out previously, ${ }^{12}$ to estimate kinetic rates it is not necessary to dissect the adhesion probability into probability distribution of point attachments. However, knowing that the measured adhesions are mediated by isolated quantal binding units that form and dissociate sequentially validates the use of the probabilistic kinetic formulation and allows a proper interpretation of the estimated rate constants.

\section{Comparison With Existing Data}

In our previous studies, the systems were limited to the Ig super gene family of molecules, i.e., $\mathrm{Fc} \gamma \mathrm{R} / \mathrm{IgG}$ interactions. ${ }^{12,13,54-56}$ The present work has tested the applicability of our micropipette method to a distinct family of molecules, i.e., E-selectin interacting with carbohydrate ligands. The kinetics of E-selectin dissociating from their carbohydrate ligands has previously been measured by using the flow chamber technique $e^{2,18,42}$ and microcantilever ${ }^{47}$ experiments. The $k_{r}^{0}$ values listed in Table 1, measured in the absence of tensile force with the micropipette using a purified E-selectin Lec-EGF construct and HL-60 and Colo-205 cells, are in good agreement with the published reverse rates at low force or extrapolated to zero force: $0.5 \mathrm{~s}^{-1}$ for endothelial cell E-selectin interacting with ligands on neutrophils, ${ }^{18} 0.7$ and $2.6 \mathrm{~s}^{-1}$ for recombinant E-selectin purified from $\mathrm{CHO}$ cells interacting with neutrophil ligands, ${ }^{2,42}$ and 0.72 and $2.2 \mathrm{~s}^{-1}$ for an Ig chimeric E-selectin construct interacting with purified $\mathrm{sLe}^{x}{ }^{47}$ In the micropipette experiments, two cells are impinged normally onto each other for contact times ranging from 0.5 to $16 \mathrm{~s}$. In the flow chamber experiments, by comparison, cells collide with the chamber floor tangentially for encounter duration as brief as milliseconds. The agreement between the zero-force reverse rates for similar biological materials measured using two distinct techniques indicates that these kinetic rates are independent of the transport processes by which the two reacting molecules are brought together and apart.

We found that the HL-60 and Colo-205 cells bound to E-selectin coated RBCs with similar cellular forward rates but dissociated twice as fast from the former as from the latter. This finding is consistent with the report that HL-60 cells only express sLe ${ }^{x}$ but Colo-205 cells express both $\mathrm{sLe}^{x}$ and $\mathrm{sLe}^{a}$, which decorate as least four different glycoproteins. ${ }^{24,46,49}$ (Because the E-selectin ligands have yet to be determined, these are treated as a single species here. Approximating the concurrent binding of multiple species with a single-species model will introduce a component into the standard errors that is not due to experimental variability, but rather is derived from the mathematical discordance between the single-species model and the potentially more complex multiple-species behavior. ${ }^{58}$ However, using the single-species approximation will not negate the discussion here.) Different ligands likely lead to different sets of $k_{f}^{0}$ and $k_{r}^{0}$ values. The similar $m_{l} A_{c} k_{f}^{0}$ values found here may be a result of different $m_{l}$, or different $A_{c}$ due to cell surface features 
such as roughness and deformability, ${ }^{56}$ which may compensate the different $k_{f}^{0}$, if any. On the other hand, it seems intuitively reasonable that dissociation of bound ligands does not depend on any of these factors. Thus, differences in the ligands are readily revealed by the different $k_{r}^{0}$ values.

We previously measured the 2D equilibrium affinities of the E-selectin/ligand binding by a centrifugation method. ${ }^{38}$ The effective affinities for Colo-205 and HL-60 cells, estimated by extrapolating the $m_{l} A_{c} K_{a}$ versus centrifugal force data to zero force, are an order of magnitude smaller than the values found here. In the present micropipette experiment, the E-selectin coated $\mathrm{RBC}$ was impinged onto the target cell to make a large contact in order to visualize multiple point attachments. In the centrifugation work, by contrast, the target cells remained rounded after being gently spun down by a small force $(\sim 5 \mathrm{pN})$ to the E-selectin coated surface, and it is expected that the contact area would be much smaller. As we have recently shown, the value of effective affinity can be dramatically altered by the contact area, ${ }^{56}$ which we hypothesize as the reason for the discrepancy between the present $m_{l} A_{c} K_{a}^{0}$ values and those previously reported.

\section{Forward Rates of E-Selectin/Ligand Interactions Are Indeed Very Rapid}

Perhaps the most variable new information generated from the present work is the effective cellular forward rates per unit density of E-selectin, $m_{l} A_{c} k_{f}^{0}$, of E-selectin/ligand interactions (Table 1). It is interesting to compare these values to those of $\mathrm{Fc} \gamma \mathrm{R} / \mathrm{IgG}$ interactions, which were measured using the same micropipette adhesion frequency assay. The 2D effective forward rates of $\mathrm{K} 562 \mathrm{Fc} \mathrm{RIII} \mathrm{b}^{\mathrm{NA} 2}$ for $\mathrm{hIgG}$ isotypes 1 and 2 are, respectively, $A_{c} k_{f}^{0}=2.8$ and $0.39 \times 10^{-7} \mu \mathrm{m}^{4} \mathrm{~s}^{-1}, 54$ which, after multiplying the surface density of Fc $\gamma \mathrm{RIIIb}$ on human neutrophils $\left(135,000\right.$ molecules per cell ${ }^{41}$ of $217 \mu \mathrm{m}^{2}$ apparent surface area $\left.{ }^{48}\right)$, convert to $m_{r} A_{c} k_{f}^{0}$ $=1.7$ and $0.24 \times 10^{-4} \mu \mathrm{m}^{2} \mathrm{~s}^{-1}$ per unit density of hIgG1 and $\mathrm{hIgG} 2$, respectively. These values are 3 or 4 orders of magnitude smaller than those listed in Table 1, suggesting that neutrophils (assuming their respective E-selectin and $\mathrm{Fc} \gamma \mathrm{RIII}^{\mathrm{NA} 2}$ binding kinetic properties are similar to those of HL-60 and K562 cells, respectively) would bind to cell-bound E-selectin 3 or 4 orders of magnitude faster than to a cell opsonized with equal density of $\operatorname{IgG} 1$ or $\operatorname{IgG} 2$, respectively. It has long been proposed that selectin/ligand binding must have rapid kinetics in order for these interactions to mediate leukocyte tethering to and rolling on endothelial cells in shear flow. Previously, surface plasmon resonance measurements of 3D forward rates of soluble P- and L-selectins for physiological ligands were used to support this hypothesis, ${ }^{27,31}$ despite the fact that their relevance to the $2 \mathrm{D}$ binding has yet to be shown, as discussed in the Introduction. The present micropipette data indicate that the E-selectin/ligand interactions are indeed of much more rapid forward rates compared to $\mathrm{Fc} \gamma \mathrm{R} / \mathrm{IgG}$ interactions. Comparing the calculation in the Introduction with the measured parameters confirms that these forward rates are sufficient. Indeed, multiplying the $m_{l} A_{c} k_{f}^{0}$ values in Table 1 with the E-selectin density of $370 \mu \mathrm{m}^{-2}$ on activated endothelial cells yields a cellular forward rate of $50 \mathrm{~s}^{-1}$. Even if the contact area $A_{c}$ is an order of magnitude smaller under flow than in the micropipette experiment, a $5 \mathrm{~s}^{-1}$ cellular forward rate would initiate binding of HL-60 or Colo-205 cells once every 200 collisions (each of millisecond duration).

\section{Issues Associated With Low Receptor Density}

As a result of the rapid forward rates and high binding affinities of the 2D E-selectin/ligand interactions, only a few E-selectin molecules per micron square of $\mathrm{RBC}$ surface is sufficient for the adhesion probability to be in the midrange, the range in which the micropipette adhesion frequency assay is designed to work. By comparison, the $\mathrm{Fc} \gamma \mathrm{R} / \mathrm{IgG}$ interactions require hundreds to thousands of interacting molecules per micron square of contact area to achieve sufficient levels of binding. ${ }^{12,13,54-56}$ Several issues have been found to associate with the present low E-selectin density condition.

To identify the rate-limiting factor(s) it is useful to decompose the binding process into a transport and a reaction step. When molecular densities are high, adhesion is likely limited by reaction because receptors would be surrounded by ligands once the two cells are in contact. When molecular densities are low, by contrast, adhesion may be limited by transport because the interacting molecules are likely far apart. However, in most 2D binding cases the receptors and ligands are brought together by the cells to which they are respectively anchored rather than diffusion as in the $3 \mathrm{D}$ case. In the micropipette experiment, such a "convective transport" process is much faster than molecular diffusion on the cell membrane if a receptor happens to be placed to the vicinity of a ligand, but completely ineffective if not. Just as the rate of a diffusion-limited reaction is influenced by the diffusion coefficient, here the question is whether the measured adhesion probability truly represents the likelihood of forming a bond between a receptor/ligand pair or merely reflects the chance of placing them sufficiently close for binding to become possible. Obviously, should the latter be the case the measured $P_{a}$ data would no longer contain the desired kinetic information. That the former still dominates the measured $\langle n\rangle / m_{r}$ vs $t$ curves is supported by the fact that reasonable kinetic rates could be extracted from the data 


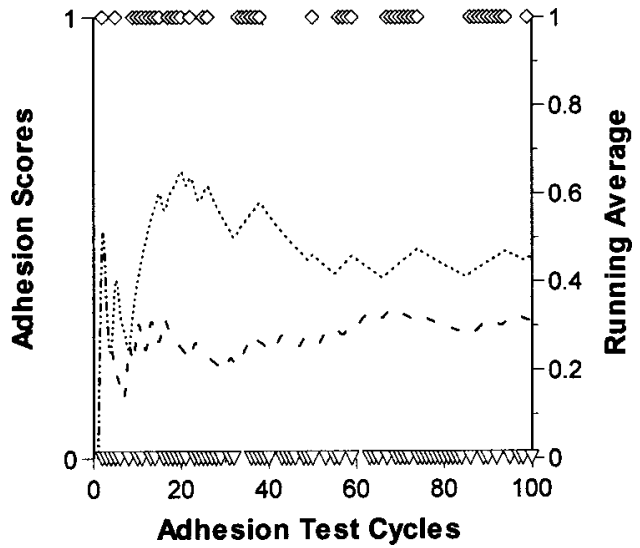

FIGURE 6. Effect of molecular density as revealed by plots of adhesion scores (points, left ordinate) and their running average (curves, right ordinate) vs test cycle counts (abscissa). Sequential contacts of a RBC coated with $5.2 \mu \mathrm{m}^{-2}$ of E-selectin with a ligand-expressing HL-60 cell resulted in clusters of adhesion events ( $\diamond$ and solid curve). By comparison, repeated touches of a RBC coated with $980 \mu \mathrm{m}^{-2}$ of hlgG2 with a CHO cell expressing $1600 \mu \mathrm{m}^{-2} \mathrm{Fc}_{\mathrm{R}} \mathrm{RIIb}^{\mathrm{NA2}}$ yielded uniformly random adhesion events ( $\nabla$ and dashed curve). For the sake of clarity, positive adhesion scores (ones) are shown for the E-selectin/ligand interaction and negative adhesion scores (zeros) are shown for the $\mathrm{Fc} \gamma \mathrm{R} I \mathrm{Il} \mathrm{b}^{\mathrm{NA} 2} / \mathrm{hlg}$ G2 interaction.

shown in Fig. 5, presumably because of the sufficiently high ligand density that compensates the low E-selectin density. However, the following observations suggest that the latter may have some contribution.

Previously, we found that adhesion events were usually uniformly distributed in the hundreds of test cycles performed on each cell pair, suggesting that whether one test resulted in an adhesion or not would not affect the likelihood for an adhesion to occur in the next test. ${ }^{12}$ In the present work, by contrast, concentrations of adhesion events were observed more frequently, as exemplified in Fig. 6. A plausible interpretation for the concentration of adhesion (or nonadhesion) events may be that the E-selectin molecules happened to be placed near by (or far away from) the ligands. Because the cells were brought into contact by the precise movement of a piezoelectric translator, approximately the same surface elements would touch each other in a series repeated contacts, leading to a correlation of the positive (or negative) adhesion scores.

The fact that the experimenter did not know whether or not interacting molecules were placed in close proximity gave rise to another uncontrollable random element to the stochastic binding process. This added randomness may explain the much higher level of data scattering (cf. Fig. 5) than seen previously. ${ }^{5-56}$ This argument is supported by an analysis of residues, which revealed twice the variance (for both the HL-60 and Colo-205 data) than seen previously. ${ }^{54}$ It is interesting to note that, com- pared to the HL-60 case, the more scattered Colo-205 data correlate with a higher effective cellular affinity $m_{l} A_{c} K_{a}^{0}$ per E-selectin density that requires a lower E-selectin density $m_{r}$ to achieve the same level of adhesion frequency (cf. Table 1). This kind of data variation could also be augmented by the presence of multiple species of carbohydrate ligands possessing different binding properties, as previously discussed.

Approximating the solution of the master equations by the Poisson distribution may also contribute some error. Unlike the previous studies, the condition for such an approximation to be valid - the bond number is sufficiently low such that the numbers of the receptors and ligands remain essentially constant in the contact area-is only marginally satisfied. Although we did not use the exact solution of the master equations to fit the data-their variation does not seem to justify the use of a more sophisticated model — we did acquire a larger size of samples to ensure their statistical reliability. In the present work, the two sets of kinetic parameters listed in Table 1 were, respectively, estimated from 49 (for HL-60 data) and 63 (for Colo-205 data) pairs cells of 100 tests each, $\sim 3$ times those normally used in the study of a $\mathrm{Fc} \gamma \mathrm{R} / \mathrm{IgG}$ interaction.

To conclude, the successful application of the micropipette method to the study of E-selectin/carbohydrate ligand interactions and its extension to the kinetic measurement of point attachments have provided further validation not only to this useful experimental tool but also to its underlying mathematical framework-the probabilistic kinetics theory. The kinetic data so obtained further our understanding of E-selectin/ligand binding and provide a basis for quantitative descriptions of interactions between flowing cells and the vessel wall under physiological conditions.

\section{ACKNOWLEDGMENTS}

The authors thank Rodger P. McEver for the generous gifts of the P-selectin construct and the PL1, ES1, and ES2 mAbs. The authors also thank Tom Williams for providing the $\mathrm{Fc} \gamma \mathrm{RIIIb} / \mathrm{hIgG} 2$ interaction data, Warren Marcus for the area-attachment experiment, and Jian Qing and Xin-Ping Li for assisting image acquisition. The manuscript was written while one of the authors (C.Z.) was on leave in Marseille, France, under the support of an INSERM Poste Orange. One of the authors (C.Z.) is indebted to Pierre Bongrand for many helpful discussions. This work was supported by NSF Grant No. BCS9350370 and NIH Grant No. AI44902 (C.Z.) as well as by NSFC Grant Nos. 10042001 and 10072071 and a TRAPOYT Award of China (M.L.). One of the authors (M.L.) is a recipient of a senior scholarship from the Ministry of Education of China. 


\section{REFERENCES}

${ }^{1}$ Alon, R., D. A. Hammer, and T. A. Springer. Lifetime of the P-selectin-carbohydrate bond and its response to tensile force in hydrodynamic flow. Nature (London) 374:539-542, 1995.

${ }^{2}$ Alon, R., S. Chen, K. D. Puri, E. B. Finger, and T. A. Springer. The kinetics of L-selectin tethers and the mechanics of selectin-mediated rolling. J. Cell Biol. 138:1169-1180, 1997.

${ }^{3}$ Araki, M., K. Araki, L. Biancone, I. Stamenkovic, S. Izui, K. Yamamura, and P. Vassalli. The role of E-selectin for neutrophil activation and tumor metastasis in vivo. Leukemia 11 Suppl. (3): 209-212, 1997.

${ }^{4}$ Asa, D., L. Raycroft, L. Ma, P. A. Aeed, P. S. Kaytes, A. P. Elhammer, and J. G. Geng. The P-selectin glycoprotein ligand functions as a common human leukocyte ligand for $\mathrm{P}$ and E-selectins. J. Biol. Chem. 270:11662-11670, 1995.

${ }^{5}$ Bell, G. I. Models for the specific adhesion of cells to cells. Science 200:618-627, 1978.

${ }^{6}$ Bell, G. I., M. Dembo, and P. Bongrand. Cell adhesion: Competition between nonspecific repulsion and specific bonding. Biophys. J. 45:1051-1064, 1984.

${ }^{7}$ Biancone, L., M. Araki, K. Araki, P. Vassalli, and I. Stamenkovic. Redirection of tumor metastasis by expression of E-selectin in vivo. J. Exp. Med. 183:581-587, 1996.

${ }^{8}$ Capo, C., F. Garrouste, A.-M. Benoliel, P. Bongrand, A. Ryter, and G. Bell. Concanavalin-A-mediated thymocte agglutination: A model for a quantitative study of cell adhesion. J. Cell. Sci. 56:21-48, 1982.

${ }^{9}$ Chen, S., R. Alon, R. C. Fuhlbrigge, and T. A. Springer. Rolling and transient tethering of leukocytes on antibodies reveal specializations of selectins. Proc. Natl. Acad. Sci. U.S.A. 94:3172-3177, 1997.

${ }^{10}$ Chen, S., and T. A. Springer. An automatic braking system that stabilizes leukocyte rolling by an increase in selectin bond number with shear. J. Cell Biol. 94:185-200, 1999.

${ }^{11}$ Chesla, S. E., and C. Zhu. Validation and accuracy assessment of the micropipet piconewton force transducer. In: 1996 Advances in Bioengineering, edited by S. Rastegar. New York: ASME, 1996, BED-Vol. 30, pp. 77 and 78.

${ }^{12}$ Chesla, S. E., P. Selvaraj, and C. Zhu. Measuring twodimensional receptor-ligand binding kinetics with micropipette. Biophys. J. 75:1553-1572, 1998.

${ }^{13}$ Chesla, S. E., P. Li, S. Nagarajan, P. Selvaraj, and C. Zhu. The membrane anchor influences ligand binding 2D kinetics rates and 3D affinity of Fc $\gamma$ RIII (CD16). J. Biol. Chem. 275:10235-10246, 2000.

${ }^{14}$ Dumaswala, U. J., M. J. Wilson, T. Jose, and D. L. Daleke. Glutamine- and phosphate-containing hypotonic storage media better maintain erythrocyte membrane physical properties. Blood 88:697-704, 1996.

${ }^{15}$ Erbe, D. V., B. A. Wolitzky, L. G. Presta, C. R. Norton, R. J. Ramos, D. K. Burns, J. M. Rumberger, B. N. N. Rao, C. Foxall, B. K. Brandley, and L. A. Lasky. Identification of an E-selectin region critical for carbohydrate recognition and cell adhesion. J. Cell Biol. 119:215-227, 1992.

${ }^{16}$ Jones, W. M., G. M. Watts, M. K. Robinson, D. Vestweber, and M. A. Jutila. Comparison of E-selectin-binding glycoprotein ligands on human lymphocytes, neutrophils, and bovine gamma delta T cells. J. Immunol. 159:3574-3583, 1997.

${ }^{17}$ Kansas, G. S. Selectins and their ligands: Current concepts and controversies. Blood 88:3259-3287, 1996.

${ }^{18}$ Kaplanski, G., C. Farnarier, O. Tissot, A. Pierres, A.-M. Benoliel, M.-C. Alessi, S. Kaplanski, and P. Bongrand.
Granulocyte-endothelium initial adhesion: Analysis of transient binding events mediated by E-selectin in a laminar show flow. Biophys. J. 64:1922-1933, 1993.

${ }^{19}$ Lasky, L. A. Selectin-carbohydrate interactions and the initiation of the inflammatory response. Annu. Rev. Biochem. 64:113-139, 1995.

${ }^{20}$ Lawrence, M. B., and T. A. Springer. Leukocytes roll on a selectin at physiological flow rates: Distinction from and prerequisite for adhesion through integrins. Cell 65:859-873, 1991.

${ }^{21}$ Li, S.-H., D. K. Burns, J. M. Rumberger, D. H. Presky, V. L. Wilkinson, M. Anostario, Jr., B. A. Wolitzky, C. R. Norton, P. C. Familletti, K. J. Kim, A. L. Goldstein, D. C. Cox, and K.-S. Huang. Consensus repeat domains of E-selectin enhance ligand binding. J. Biol. Chem. 269:4431-4437, 1994.

${ }^{22}$ Liu, W.-J., V. Ramachandran, J. Kang, T. K. Kishimoto, R. D. Cummings, and R. P. McEver. Identification of N-terminal residues on P-selectin glycoprotein ligand-1 required for binding to P-selectin. J. Biol. Chem. 2733:7078-7087, 1998.

${ }^{23}$ Long, M., H. L. Goldsmith, D. F. J. Tees, and C. Zhu. Probabilistic modeling of shear-induced formation and breakage of doublets cross linked by receptor-ligand bonds. Biophys. J. 76:1112-1128, 1998.

${ }^{24}$ Majuri, M.-L., P. Mattila, and R. Renkonen. Recombinant E-selectin-protein mediates tumor cells adhesion via sialylLea and sialyl-Lex. Biochem. Biophys. Res. Commun. 182:1376-1382, 1992.

${ }^{25}$ McEver, R. P., K. L. Moore, and R. D. Cummings. Leukocyte trafficking mediated by selectin-carbohydrate interactions. J. Biol. Chem. 270:11025-11028, 1995.

${ }^{26}$ McQuarrie, D. A. Kinetics of small systems. I. J. Phys. Chem. 38:433-436, 1963.

${ }^{27}$ Mehta, P., R. D. Cummings, and R. P. McEver. Affinity and kinetic analysis of $\mathrm{P}$-selectin binding to $\mathrm{P}$-selectin glycoprotein ligand-1. J. Biol. Chem. 273:32506-32513, 1998.

${ }^{28}$ Mehta, P., K. D. Patel, T. M. Laue, H. P. Erickson, and R. P. McEver. Soluble monomeric P-selectin containing only the lectin and epidermal growth factor domains binds to P-selectin glycoprotein ligand-1 on leukocytes. Blood 90:2381-2389, 1997.

${ }^{29}$ Merkel, R., P. Nassoy, A. Leung, K. Ritchie, and E. Evans. Energy landscapes of receptor-ligand bonds explored with dynamic force spectroscopy. Nature (London) 397:50-53, 1999.

${ }^{30}$ Moore, K. L., K. D. Patel, R. E. Bruehl, L. Fugang, D. A. Johnson, H. S. Lichenstein, R. D. Cummings, D. F. Bainton, and R. P. McEver. P-selectin glycoprotein ligand-1 mediates rolling of human netrophils on P-selectin. J. Cell Biol. 128:661-671, 1995.

${ }^{31}$ Nicholson, M. W., A. N. Barclay, M. S. Singer, S. D. Rosen, and P. A. van der Merwe. Affinity and kinetic analysis of L-selectin (CD62L) binding to glycosylation-dependent celladhesion molecule-1. J. Biol. Chem. 273:763-770, 1998.

${ }^{32}$ Nelson, R. M., A. Venot, M. P. Bevilacqua, R. J. Linhardt, and I. Stamenkovic. Carbohydrate-protein interactions in vascular biology. Annu. Rev. Cell Dev. Biol. 11:601-631, 1995.

${ }^{33}$ Patel, K. D., K. L. Moore, M. U. Nollert, and R. P. McEver. Neutrophils use both shared and distinct mechanisms to adhere to selectins under static and flow conditions. J. Clin. Invest. 96:1887-1896, 1995.

${ }^{34}$ Pierres, A., A. M. Benoliel, P. Bongrand, and P. A. van der Merwe. Determination of the lifetime and force dependence of interactions of single bonds between surface-attached CD2 and CD48 adhesion molecules. Proc. Natl. Acad. Sci. U.S.A. 93:15114-15118, 1996. 
${ }^{35}$ Pierres, A., A.-M. Benoliel, P. Bongrad, and P. A. van der Merwe. The dependence of the association rate of surfaceattached adhesion molecules CD2 and CD48 on separation distance. FEBS Lett. 403:239-244, 1997.

${ }^{36}$ Pierres, A., H. Feracci, V. Delmas, A. M. Benoliel, J. P. Thiery, and P. Bongrand. Experimental study of the interaction range and association rate of surface-attached cadherin 11. Proc. Natl. Acad. Sci. U.S.A. 95:9256-9261, 1998.

${ }^{37}$ Pierres, A., A.-M. Benoliel, and P. Bongrad. Studying receptor-mediated cell adhesion at the single molecule level. Cell Adhes. Commun. 5:375-395, 1998.

${ }^{38}$ Piper, J. W., R. A. Swerlick, and C. Zhu. Determining force dependence of two-dimensional receptor-ligand binding affinity by centrifugation. Biophys. J. 74:492-513, 1998.

${ }^{39}$ Ramachandran, V., M. U. Nollert, H.-Y. Qiu, W.-J. Liu, R. D. Cummings, C. Zhu, and R. D. McEver. Tyrosine replacement in P-selectin glycoprotein ligand-1 affects distinct kinetic and mechanical properties of bonds with P- and L-selectin. Proc. Natl. Acad. Sci. U.S.A. 96:13771-13776, 1999.

${ }^{40}$ Selvaraj, P., M. L. Dustin, R. Mitnacht, T. Hunig, T. A. Springer, and M. L. Plunkett. Rosetting of human T lymphocytes with sheep and human erythrocytes. Comparison of human and sheep ligand binding using purified $\mathrm{E}$ receptor. $J$. Immunol. 139:2690-2695, 1987.

${ }^{41}$ Selvaraj, P., W. F. Rosse, R. Silber, and T. A. Springer. The major Fc receptor in blood has a phosphatidylinositol anchor and is deficient in paroxysmal nocturnal haemoglobinuria. Nature (London) 333:565-567, 1988.

${ }^{42}$ Smith, M. J., E. L. Berg, and M. B. Lawrence. A direct comparison of selectin-mediated transient, adhesive events using high temporal resolution. Biophys. J. 77:3371-3383, 1999.

${ }^{43}$ Springer, T. A. Traffic signals on endothelium for lymphocyte recirculation and leukocyte emigration. Annu. Rev. Physiol. 57:827-872, 1995.

${ }^{44}$ Steegmaier, M., A. Levionovitz, S. Isenmann, E. Borges, M. Lenter, H. P. Kocher, B. Kleuser, and D. Vestweber. The E-selectin-ligand ESL-1 is a variant of a receptor for fibroblast growth factor. Nature (London) 373:615-620, 1995.

${ }^{45}$ Swift, D. G., R. G. Posner, and D. A. Hammer. Kinetics of adhesion of IgE-sensitized rat basophilic leukemia cells to surface-immobilized antigen in Couette flow. Biophys. J. 75:2597-2611, 1998.

${ }^{46}$ Takada, A., K. Ohmori, T. Yoneda, K. Tsuyuoka, A. Hasegawa, M. Kiso, and R. Kannagi. Contribution of carbohydrate antigens sialyl Lewis A and sialyl Lewis X to adhesion of human cancer cells to vascular endothelium. Cancer Res. 53:354-361, 1993

${ }^{47}$ Tees, D. F., R. E. Waugh, and D. A. Hammer. A microcantilever device to assess the effect of force on the lifetime of selectin-carbohydrate bonds. Biophys. J. 80:668-682, 2001.
${ }^{48}$ Ting-Beall, P., D. Needham, and R. M. Hochmuth. Volume and osmotic properties of human neutrophils. Blood 81:2774-2780, 1993.

${ }^{49}$ Tomlinson, J., J. L. Wang, S. H. Barsky, M. C. Lee, J. Bischoff, and M. Nguyen. Human colon cancer cells express multiple glycoprotein ligands for E-selectin. Int. J. Oncol. $16: 347-353,2000$.

${ }^{50}$ Ushiyama, S., T. M. Laue, K. L. Moore, H. P. Erickon, and R. D. McEver. Structural and functional characterization of monomeric soluble P-selectin and comparison with membrane P-selectin. J. Biol. Chem. 268:15229-15237, 1993.

${ }^{51}$ Varki, A. Selectin ligands. Proc. Natl. Acad. Sci. U.S.A. 91:7390-7397, 1994.

${ }^{52}$ Varki, A. Selectin ligands: Will the real ones please stand up? J. Clin. Invest. 99:158-162, 1997.

${ }^{53}$ von Andrian, U. H., J. D. Chambers, L. M. McEvoy, R. F. Bargatze, K. E. Arfors, and E. C. Butcher. Two-step model of leukocyte-endothelial cell interactions in inflammation: Distinct roles for LECAM- 1 and the leukocyte $\beta 2$-integrins in vivo. Proc. Natl. Acad. Sci. U.S.A. 88:7538-7542, 1991.

${ }^{54}$ Williams, T. E., P. Selvaraj, and C. Zhu. Concurrent binding to multiple ligands: Kinetic rates of $\mathrm{CD} 16 \mathrm{~b}$ for membranebound IgG1 and IgG2. Biophys. J. 79:1858-1866, 2000.

${ }^{55}$ Williams, T. E., S. Nagarajan, P. Selvaraj, and C. Zhu. Concurrent and independent binding of $\mathrm{Fc} \gamma$ receptors IIa and IIIb to surface-bound IgG. Biophys. J. 79:1867-1875, 2000.

${ }^{56}$ Williams, T. E., S. Nagarajan, P. Selvaraj, and C. Zhu. Quantifying the impact of membrane microtopology on effective two-dimensional affinity. J. Biol. Chem. 276:13283-13288, 2001.

${ }^{57}$ Zaifert, K., and M. C. Cohen. Colo-205 utilizes E-selectin to adhere to human endothelium. Clin. Immun. Immunopath. 68:51-56, 1993.

${ }^{58}$ Zhu, C., and T. E. Williams. Modeling concurrent binding of multiple molecular species in cell adhesion. Biophys. J. 79:1850-1857, 2000.

${ }^{59}$ Zhu, C., M. Long, and P. Bongrand. Measuring receptor/ ligand interaction at the single bond level: Experimental and interpretative issues. Ann. Biomed. Eng. (in press).

${ }^{60}$ Zöllner, O., M. C. Lenter, J. E. Blanks, E. Borges, M. Steegmaier, H. G. Zerwes, and D. Vestweber. L-selectin from human, but not from mouse neutrophils binds directly to E-selectin. J. Cell Biol. 136:707-716, 1997.

${ }^{61}$ See AIP Document No. E-PAPS: ABMECF-029-010111 for a movie file showing the distinction between point and area attachments in separation processes. EPAPS document files may be retrieved free of charge from AIP's FTP server (http://www.AIP.org/pubservs/paps.html) or from ftp.aip.org in the directory /epaps/. For further information, e-mail: paps@aip.org or fax 516-576-2223. 